\title{
Long-Wavelength Phonon Scattering in Nonpolar Semiconductors
}

\author{
Lawætz, Peter
}

Published in:

PHYSICAL REVIEW

Link to article, DOI:

10.1103/PhysRev.183.730

Publication date:

1969

Document Version

Publisher's PDF, also known as Version of record

Link back to DTU Orbit

Citation (APA):

Lawætz, P. (1969). Long-Wavelength Phonon Scattering in Nonpolar Semiconductors. PHYSICAL REVIEW, 183(3), 730-739. https://doi.org/10.1103/PhysRev.183.730

\section{General rights}

Copyright and moral rights for the publications made accessible in the public portal are retained by the authors and/or other copyright owners and it is a condition of accessing publications that users recognise and abide by the legal requirements associated with these rights.

- Users may download and print one copy of any publication from the public portal for the purpose of private study or research.

- You may not further distribute the material or use it for any profit-making activity or commercial gain

- You may freely distribute the URL identifying the publication in the public portal

If you believe that this document breaches copyright please contact us providing details, and we will remove access to the work immediately and investigate your claim. 
When more than one phonon is emitted in a cascade process, however, the phonon energy becomes less precise, and the transition takes place further from. $\mathbf{k}=0$. We have shown by a qualitative argument, taking into consideration the curvature of the valence band and the dispersion curves for the LO phonon, that the energy spacing of the higher-energy minima will gradually decrease, and that fine structure will no longer be discernible when several phonons are emitted in cascade. This is borne out exactly by our experimental measurements.

Finally, certain features which are observed in the one-phonon region of the photoconductivity spectrum are considered to be due to hole capture, with the emission of one phonon, to excited states of the acceptor center to which transitions from the ground state are forbidden. This interpretation is reinforced by the fact that the energy of one of these forbidden transitions is in good agreement with other theoretical and experimental evidence.

\section{ACKNOWLEDGMENTS}

We should like to thank Gordon Davies for helpful discussions concerning the interpretation of the data and for preparing the computer programs. The diamonds were provided by Dr. F. A. Raal and Dr. L. du Preez of the Diamond Research Laboratory, Johannesburg, and we are grateful to them for their cooperation and their continued interest in this work. We are also indebted to Dr. W. J. Choyke of the Westinghouse Research Laboratories for providing the $\mathrm{Au}-\mathrm{Ta}-\mathrm{Al}$ alloy used for welding $p$-type contacts to the diamonds. The project was partially financed by equipment grants from Industrial Distributors Ltd., the Science Research Council, and the Royal Society.

\title{
Long-Wavelength Phonon Scattering in Nonpolar Semiconductors
}

\author{
P. LaWAetZ \\ Physics Laboratory III, The Technical University of Denmark, Lyngby, Denmark
}

(Received 14 October 1968)

\begin{abstract}
The long-wavelength acoustic- and optical-phonon scattering of carriers in nonpolar semiconductors is considered from a general point of view. The deformation-potential approximation is defined and it is shown that long-range electrostatic forces give a nontrivial correction to the scattering. For completeness, corrections due to nonlocal effects like spin-orbit coupling are treated, but the general conclusion is that the deformation-potential approach is a very good approximation except in some cases of optical-phonon scattering. The smallness of the nontrivial corrections is interpreted in terms of the very-short-range nature of interactions in a covalent semiconductor.
\end{abstract}

\section{INTRODUCTION}

$I^{\mathrm{T}}$ $T$ has recently been suggested that the usual deformation-potential theory may be inadequate for the description of long-wavelength phonon scattering in p-type Ge. ${ }^{1}$ Before that, theoretical investigations of the phonon spectrum in Ge had shown ${ }^{2}$ that long-range forces of quadrupole nature are needed for the interpretation of the experimental data. Tolpygo ${ }^{3}$ found that these electrostatic forces also give a nontrivial contribution to the electron-phonon interaction, and a quantitative estimate ${ }^{4}$ for Ge indicated that the effect may be

1 P. Lawaetz, Phys. Rev. 174, 867 (1968)

${ }^{2} \mathrm{M}$. Lax, in Proceedings of the International Conference on Lattice Dynamics, Copenhagen, 1963, edited by R. F. Wallis (Pergamon Press, Inc., New York, 1965), p. 179. This paper contains references to earlier work on the theory of the phonon spectra in nonpolar semiconductors.

${ }^{3}$ K. B. Tolpygo, Fiz. Tverd. Tela 4, 1765 (1962) [English transl.: Soviet Phys.-Solid State 4, 1297 (1963)].

$4 \mathrm{Z}$. A. Demidenko and K. B. Tolpygo, Fiz. Tverd. Tela 6, 3321 (1964) [English transl.: Soviet Phys.-Solid State 6, 2656 (1965)]. of the same order of magnitude as that derived from deformation-potential theory.

In order to study this and other possible shortcomings of deformation-potential theory, we shall begin in Sec. II by establishing the theoretical background of this model of the interaction between carriers and longwavelength acoustic phonons. The long-range interaction is then introduced in Sec. III and Tolpygo's results ${ }^{3}$ are rederived, but on a new and more general basis, and new features arise as a consequence of the combined treatment.

After these general considerations, we shall be concerned quantitatively in Sec. IV with the electrostatic contribution to the long-wavelength acoustic scattering in Ge. In the case of electrons, the new terms are readily incorporated in the theory of Herring and Vogt, ${ }^{5}$ and it is shown that the new theory may be consistent with experimental data if the effect is of the estimated magnitude or even somewhat larger. For

\footnotetext{
${ }^{5}$ C. Herring and E. Vogt, Phys. Rev. 101, 944 (1956)
} 
holes, the expressions for the scattering rates given in Ref. 1 are extended to include the extra terms. Results of calculations indicate that the fit to experimental data is not improved over that obtained previously ${ }^{1}$ without the extra contribution.

A deformation-potential theory of long-wavelength optical-phonon scattering can also be formulated. ${ }^{6,7}$ The validity of this model is investigated in Sec. V, and the effect of the long-range electrostatic interaction is shown to be nontrivial. This contribution is nonzero even for those band extrema where the usual optical deformation-potential constant ${ }^{8}$ vanishes by symmetry. ${ }^{9}$ This means that the relevant scattering mechanisms ${ }^{10}$ in $n$-Si should be reconsidered. Also, interesting corrections to the usual model of optical-phonon scattering ${ }^{1}$ in $p$-Ge are discussed. This mechanism may explain the discrepancy between experimental data on galvanomagnetic effects at room temperature and existing theory. ${ }^{1}$

For the sake of completeness, we briefly discuss the effects of spin-orbit coupling in Sec. VI. In the case of static strain, it has been shown ${ }^{11}$ that such effects are included in the usual deformation-potential constants. For acoustic-phonon scattering, however, a nontrivial correction to the scattering potential is found. Nevertheless, spin-orbit effects are usually small and the above effect is probably of no practical importance.

A discussion of physical reasons for the smallness of the electrostatic effects is contained in Sec. VII. A short-range interaction model is proposed and is shown to be in qualitative agreement with our general knowledge of nonpolar semiconductors.

\section{DEFORMATION POTENTIAL}

Seitz ${ }^{12}$ initiated the study of the acoustic-phonon scattering in nonpolar semiconductors by considering carriers in a simple spherical band. Using the deformableion model, ${ }^{13}$ he found that the matrix element of the interaction could be expressed by a single parameter. Bardeen and Shockley ${ }^{14}$ later interpreted this parameter in terms of the displacement of the band due to a dilatation of the crystal, but their so-called deformation potential was more general. The fundamental ideas behind the deformation-potential method as applied to nonpolar semiconductors are (a) that the perturbing Hamiltonian is of the same form for lattice scattering

${ }^{6}$ E. G. S. Paige, Progress in Semiconductors (Heywood and Co., Ltd., London, 1964), Vol. 8, p. 1.

${ }^{7}$ G L. Bir and G. E. Pikus, Fiz. Tverd. Tela 2, 2287 (1960) [English transl.: Soviet Phys.-Solid State 2, 2039 (1961)].

${ }^{8}$ In order to avoid confusion, the well-known parameters $\Xi_{u}, \Xi_{l}, a, b, d$, etc., will be termed "deformation-potential constants."

${ }^{9}$ W. A. Harrison, Phys. Rev. 104, 1281 (1956)

${ }^{10}$ D. Long, Phys. Rev. 120, 2024 (1960).

${ }^{11}$ G. E. Pikus and G. L. Bir, Fiz. Tverd. Tela 1, 1624 (1959)

[English transl.: Soviet Phys.-Solid State 1, 1502 (1960)].

${ }^{12}$ F. Seitz, Phys. Rev. 73, 549 (1948).

${ }^{13}$ F. Bloch, Z. Physik 52, 555 (1928).

${ }^{14}$ J. Bardeen and W, Shockley, Phys. Rev, 80, 72 (1950), as for homogeneous strain of the crystal, (b) that a long-wavelength acoustic lattice vibration may be regarded as a slowly varying, locally homogeneous strain, and (c) that therefore the matrix elements of acousticphonon scattering can be expressed in terms of the few parameters determining the change in band structure due to an external uniaxial stress. As shown in the present work, one should add the condition that the interaction must be of limited range. The breakdown of this approximation will be discussed in Sec. III.

The feature (a) was originally based on intuition, but has later been justified by Haug ${ }^{15}$ and corresponds to the so-called "static" approximation (as opposed to the adiabatic approximation). Here the electron-phonon interaction potential is simply the change in the selfconsistent one-electron potential caused by the phononinduced disturbance in the crystal structure. In the following, we are concerned only with crystals with a diamond-type lattice. Assuming that the self-consistent potential is a differentiable function of the atomic coordinates, we have as the most general form of the potential change for one-phonon processes as well as for effects linear in static strain

$$
\delta V(\mathbf{r})=\sum_{n \alpha} \mathbf{V}_{n}^{\alpha}\left(\mathbf{r}, \mathbf{R}_{n}^{\alpha}\right) \cdot \mathbf{u}_{n}{ }^{\alpha} .
$$

Here $\mathbf{r}$ is the electron coordinate, $\mathbf{R}_{n}$ the center of mass of the $n$th unit cell ( $n=0$ corresponds to the cell in which $\mathbf{r}$ is lying), and the equilibrium atomic sites $\mathbf{R}_{n}{ }^{\alpha}$ are given by

$$
\mathbf{R}_{n}^{\alpha}=\mathbf{R}_{n}+\alpha \mathbf{t}
$$

where $\alpha= \pm 1$ and $2 \mathrm{t}$ is the vector connecting the two atoms in the unit cell of the diamond lattice. Further, $\mathbf{u}_{n}{ }^{\alpha}$ is the displacement of the atom $\mathbf{R}_{n}{ }^{\alpha}$, and $\mathbf{V}_{n}{ }^{\alpha}$ are first-order expansion coefficients.

In order to investigate the validity of deformationpotential theory, we shall first establish the connection between the coefficients $\mathbf{V}_{n}{ }^{\alpha}$ and those coefficients normally appearing in the theory of the deformation potential. ${ }^{7}$ In a crystal subjected to an infinitesimal, homogeneous deformation, the displacement of the $\alpha$ th atom in the $n$th cell may be expressed as follows:

with

$$
\begin{gathered}
\mathbf{u}_{n}{ }^{\alpha}=\mathbf{u}_{1}+\alpha \mathbf{u}_{2}+\mathbf{M} \cdot\left(\mathbf{R}_{n}{ }^{\alpha}-\mathbf{R}_{0}\right), \\
\mathbf{u}_{1} \equiv \frac{1}{2}\left(\mathbf{u}_{0}{ }^{+}+\mathbf{u}_{0}^{-}\right)
\end{gathered}
$$

being the displacement of the reference point $\mathbf{R}_{0}, \mathbf{u}_{2}$ the internal strain in the cell,

and

$$
\begin{aligned}
\mathbf{u}_{2} & \equiv \frac{1}{2}\left(\mathbf{u}_{0}^{+}-\mathbf{u}_{0}^{-}\right)-\mathbf{M} \cdot \mathbf{t}, \\
M_{i j} & \equiv \varepsilon_{i j}+\frac{1}{2} \sum_{k} \epsilon_{i j k} \operatorname{curl}_{k} \mathbf{u},
\end{aligned}
$$

where $\varepsilon$ is the symmetric strain tensor and the final antisymmetric term denotes an infinitesimal rotation of the crystal. $\epsilon_{i j k}$ is the Levi-Civita symbol. It is well

\footnotetext{
${ }^{15}$ A. Haug, Z. Physik 175, 166 (1963).
} 
known that the internal strain $\mathbf{u}_{2}$ is related to the macroscopic strain $\varepsilon$ by ${ }^{16}$

$$
u_{2 i}=\gamma \sum_{j k}\left|\epsilon_{i j k}\right| \varepsilon_{j k}
$$

where $\gamma$ is a constant with the dimension of length.

Denoting the potential change due to a homogeneous deformation by $\delta V_{d}$, we obtain by insertion of the displacement (2.3) into Eq. (2.1)

$$
\delta V_{d}(\mathbf{r})=\mathbf{u}_{1} \cdot \mathbf{V}_{1}+\mathbf{u}_{2} \cdot \mathbf{V}_{2}+\sum_{i j} \varepsilon_{i j} V_{i j}^{\prime}+\operatorname{curlu} \cdot \mathbf{V}^{\prime \prime},
$$

where

$$
\begin{aligned}
\mathbf{V}_{1}(\mathbf{r}) & =\sum_{n \alpha} \mathbf{V}_{n}{ }^{\alpha}, \\
\mathbf{V}_{2}(\mathbf{r}) & =\sum_{n \alpha} \alpha \mathbf{V}_{n}{ }^{\alpha}, \\
V_{i j}^{\prime} & =\sum_{n \alpha} V_{n i}{ }^{\alpha}\left(R_{n j}-R_{0 j}\right)+V_{2 i} t_{j}, \\
V_{k}^{\prime \prime} & =\frac{1}{2} \sum_{i j} \epsilon_{i j k}\left[\sum_{n \alpha} V_{n i}{ }^{\alpha}\left(R_{n j}-R_{0 j}\right)+V_{2 i} t_{j}\right] .
\end{aligned}
$$

From infinitesimal-displacement invariance it is easily shown that

$$
\mathbf{V}_{1}(\mathbf{r})=-\nabla_{\mathbf{r}} V_{0}(\mathbf{r})
$$

in which $V_{0}(\mathbf{r})$ is the self-consistent potential of the undisturbed crystal. The internal strain $\mathbf{u}_{2}$ is equivalent to a uniform "optical" displacement, and thus $\mathbf{V}_{2}$ is in fact the optical deformation potential ${ }^{6,7}$ which will be discussed in Sec. V. Owing to the relation (2.7) the term $\mathbf{u}_{2} \cdot V_{2}$ in Eq. (2.8) may be incorporated in the $\varepsilon$ term. The last term in (2.8) vanishes ${ }^{7}$ because the potential at a certain point is invariant under a rigid rotation of the crystal about that point. Thus

$$
\mathbf{V}^{\prime \prime}(\mathbf{r})=0 \text {, }
$$

and it follows from (2.11) and (2.12) that $V_{i j}^{\prime}$ is symmetric in $i$ and $j$. It will be shown in Sec. VI that the argument leading to (2.14) is valid only for a local oneelectron potential. Corrections may, therefore, be expected from nonlocal effects like spin-orbit coupling, exchange, etc.

The potential change (2.8) now has the form

$$
\delta V_{d}(\mathbf{r})=-\mathbf{u}_{1} \cdot \nabla V_{0}+\sum_{i j} \varepsilon_{i j} V_{i j}
$$

with

$$
V_{i j}=V_{i j}^{\prime}+\gamma \sum_{k}\left|\epsilon_{i j k}\right| V_{2 k}
$$

The next step in the usual deformation-potential theory is to consider $\delta V_{d}$ in an effective-mass representation following the displacement $\mathbf{u}_{1}{ }^{7,17}$ Then the term with $\mathbf{u}_{1}$ disappears, and the new coefficients of $\varepsilon_{i j}$ are essentially matrix elements over the unit cell of the

${ }^{16}$ A. Segmüller and H. R. Neyer, Physik Kondensienten Materie 4, 63 (1965).

17 G. D. Whitfield, Phys. Rev. 121, 720 (1961). operator $^{7,17}$

$$
D_{i j}=-\left(p_{i}+\hbar k_{0 i}\right)\left(p_{j}+\hbar k_{0 j}\right) / m+V_{i j},
$$

where $\mathbf{p}$ is the momentum operator, $m$ the free-electron mass, and $\mathbf{k}_{\mathbf{0}}$ the wave vector of the band extremum in question. These matrix elements of $D_{i j}$ are the deformation-potential constants.

We now consider the potential change (2.1) from the point of view of acoustic-phonon scattering. For phonons of wave vector $\mathbf{q}$ and branch $s$, the atomic displacements $\mathbf{u}_{n}{ }^{\alpha}$ have the form

$$
\mathbf{u}_{n}{ }^{\alpha}=A_{s} e^{i \mathbf{q} \cdot \mathbf{R}_{n} \alpha} \hat{e}_{s}^{\alpha}(\mathbf{q})+\text { H.c. },
$$

where $A_{s}$ is essentially the phonon-annihilation operator and $\hat{e}_{s}$ is the polarization vector. H.c. denotes Hermitian conjugation and stands for the phonon-emission term. The treatment of this term parallels that of the absorption term and is, therefore, not considered in thefollowing.

For long wavelengths the polarization vectors may be expanded in a power series in $q$, and we recall that for the acoustic branches to first order in $q$

where $^{18}$

$$
\begin{gathered}
\hat{e}^{\alpha}(\mathbf{q})=\hat{e}_{0}(\hat{q})+\alpha q \mathbf{e}_{1}(\hat{q}), \\
e_{1 i}(\hat{q})=i \gamma \sum_{j k}\left|\epsilon_{i j k}\right| \hat{q}_{j} \hat{e}_{0 k}(\hat{q}),
\end{gathered}
$$

$\gamma$ being the same constant as in Eq. (2.7) since it does not depend on the vibrational state of the crystal. ${ }^{18}$

As a basis of deformation-potential theory, we assume that the potential change at $\mathbf{r}$ depends only upon the disturbance of the crystal in the vicinity of $\mathbf{r}$, i.e., at distances small compared to the phonon wavelength. This condition will be discussed further when we consider the electrostatic potenital. For the evaluation of (2.1) it is then valid to expand the acousticphonon displacement (2.18) in powers of $\mathbf{q} \cdot\left(\mathbf{R}_{n}{ }^{\alpha}-\mathbf{R}_{0}\right)$. Retaining zeroth-and first-order terms only, the acoustic displacements $\mathbf{u}_{n}{ }^{\alpha}$ may be expressed in the same form as (2.3), with

$$
\begin{aligned}
& \mathbf{u}_{1}{ }^{\prime}=A_{s} e^{i \mathbf{q} \cdot \mathbf{R}_{0}} \hat{e}_{0}(\hat{q}), \\
& \mathbf{u}_{2}{ }^{\prime}=A_{s} e^{i \mathbf{q} \cdot \mathbf{R}_{0}} q \mathbf{e}_{1}(\hat{q}), \\
& \varepsilon_{i j}{ }^{\prime}=A_{s} e^{i \mathbf{q} \cdot \mathbf{R}_{0} \frac{1}{2}} i q\left[\hat{q}_{i} \hat{e}_{0 j}+\hat{q}_{j} \hat{e}_{0 i}\right] .
\end{aligned}
$$

It follows directly that the interaction potential for acoustic-phonon scattering in the deformation-potential approximation is $\delta V_{d}$ determined by Eq. (2.15), with $\mathbf{u}_{1}{ }^{\prime}$ and $\varepsilon^{\prime}$ givenby (2.21) and (2.23), respectively. At this point, it is convenient for the further treatment in scattering theory ${ }^{7}$ to replace $e^{i \mathbf{q} \cdot \mathbf{R}_{0}}$ by $e^{i \mathbf{q} \cdot \mathbf{r}}$, and it is readily seen that this makes no difference to first order in $q$.

\section{ELECTROSTATIC INTERACTION}

In order to discuss the validity of the deformationpotential approach to the description of long-wave-

${ }^{18}$ H. M. J. Smith, Phil. Trans. Roy. Soc. (London) A241, 105 (1948). In this paper, the relation between $\mathbf{e}_{1}$ and $\hat{e}_{0}$ is derived from a specific model of force constants, but the result is of general validity. 
length phonon scattering, we consider the interaction potential (2.1) in a general way. By insertion of the atomic displacement (2.18), we obtain

$$
\delta V=A_{s} e^{i \mathbf{q} \cdot \mathbf{r}} \sum_{\alpha} \hat{e}_{s}^{\alpha}(\mathbf{q}) \cdot \mathbf{U}^{\alpha}(\mathbf{q}, \mathbf{r}),
$$

where $\mathbf{U}$ is essentially the Fourier transform of $\mathbf{V}_{n}$,

$$
\mathbf{U}^{\alpha}(\mathbf{q}, \mathbf{r})=\sum_{n} \mathbf{V}_{n}{ }^{\alpha} e^{i \mathbf{q} \cdot\left(\mathbf{R}_{n} \boldsymbol{\alpha}-\mathbf{r}\right)}
$$

It is now obvious that the deformation-potential method corresponds to a Taylor-series expansion in $\mathbf{q}$ of $\mathbf{U}^{\alpha}(\mathbf{q}, \mathbf{r})$, keeping terms to first order in q. The existence and convergence properties of such a series depend on the form of $\mathbf{V}_{n}{ }^{\alpha}$ as a function of $\mathbf{R}_{n}{ }^{\alpha}-\mathbf{r}$.

When $\mathbf{V}_{n}{ }^{\alpha}$ has a well-defined range $\lambda$, e.g., by exponential decrease, the Taylor series is expected to converge for $q \leqslant \lambda^{-1}$. In that case, the deformation potential is valid for small enough $q$ and any breakdown would show up as large terms of higher order in $q$. This type of correction will not be considered further because it does not seem relevant for the long-wavelength phonon scattering encountered in nonpolar semiconductors.

If part of $\mathbf{V}_{n}{ }^{\alpha}$ is electrostatic, it is readily shown that some of the expansion coefficients of $\mathbf{U}^{\alpha}(\mathbf{q}, \mathbf{r})$ are infinite, so that a Taylor series does not exist for any q. This is due to the indefinite range of electrostatic forces. In that case, the oscillatory form in (3.2) is essential, and we may then expect new effects. It will be shown that an expansion in powers of $|\mathbf{q}|$ is then appropriate with coefficients depending on $\hat{q}$. On the other hand, only electrostatic forces have this peculiar property, and so when we have included these effects, our theory of the deviations from the deformation potential is complete. ${ }^{19}$ It follows that the electrostatic contribution to the interaction potential is of particular interest.

In a nonpolar crystal with a diamond-type lattice, the displacement of an atom induces a disturbance in the surrounding charge distribution, resulting in an electric moment basically of quadrupole nature, ${ }^{2}$ but with higher pole contributions as well. Without loss of generality, these poles may be located to the equilibrium site of the displaced atom because infinitesimaldisplacement invariance and inversion symmetry will fix the contributions to $\mathbf{U}^{\alpha}$ from the cell $n=0$. This may not be rigorously true for small $n$, but such short-range phenomena are taken into account in the deformation potential. For a uniform displacement, there can be no electrostatic fields present, and so for these potentials

$$
\sum_{n \alpha} \mathbf{V}_{n}^{\alpha}=0
$$

In other words, the infinitesimal-displacement invariance ensures that the total linear electric moment of the crystal is zero. From the inversion symmetry of the

${ }^{19}$ The nonlocal corrections discussed in Sec. VI are considered as a previously neglected part of the deformation potential. $n$th unit cell we conclude that $\mathbf{V}_{n}^{+}-\mathbf{V}_{n}^{-}$must vanish at $\mathbf{r}=\mathbf{R}_{n}$ (the inversion center). It follows that for $2^{p}$-poles the moments $Q_{p}^{\alpha}$ satisfy

$$
Q_{p}^{+}=(-1)^{p-1} Q_{p}^{-} \text {. }
$$

As a direct consequence of (3.3) and (3.4), dipoles $(p=1)$ are excluded, because otherwise the sum in (3.3) is divergent for all $\mathbf{r} \neq \mathbf{R}_{\mathbf{0}}$. Furthermore, the macroscopic inversion symmetry of the crystal demands that

$$
\sum_{n \alpha} \alpha \mathbf{V}_{n}^{\alpha}=0
$$

In Appendix A it is shown that the above requirements do not exclude any moments with $p>1$, in contrast to earlier theory. ${ }^{20}$ From the discussion in Appendix A we derive the additional result that the contribution $\mathbf{V}_{0}{ }^{\alpha}$ from the cell $n=0$ is described in terms of the same poles as for $n \neq 0$.

We now consider the difference $\delta V_{e}$ between the exact interaction potential (3.1) and the deformation potential (2.15). To first order in $q$, this additional electrostatic potential takes the form

$$
\begin{aligned}
\delta V_{e} & =A_{s} e^{i \mathbf{q} \cdot \mathbf{r}}\left[\hat{e}_{0} \cdot\left(\mathbf{S}_{1}-\mathbf{S}_{2}\right)+q \mathbf{e}_{1} \cdot \mathbf{S}_{3}\right] \\
S_{1} & =\sum_{n \alpha} \mathbf{V}_{n}^{\alpha} e^{i \mathbf{q} \cdot\left(\mathbf{R}_{n}^{\alpha-r}\right)} \\
S_{2} & =\sum_{n \alpha} \mathbf{V}_{n}^{\alpha}\left[i \mathbf{q} \cdot\left(\mathbf{R}_{n}-\mathbf{R}_{0}\right)\right] \\
S_{3} & =\sum_{n \alpha} \alpha \mathbf{V}_{n}^{\alpha} e^{i \mathbf{q} \cdot\left(\mathbf{R}_{n}^{\alpha-r}\right)} .
\end{aligned}
$$

$\mathbf{S}_{1}$ and $\mathbf{S}_{3}$ give the electrostatic contribution to the scattering potential (exact for all $q$ ), whereas $\mathbf{S}_{2}$ stands for that part of the long-range electrostatic potential which has already been taken into account in the deformation potential (to first order in $q$ ).

Using a general $2^{p}$-pole potential of the form

$$
\mathbf{V}_{n i}{ }^{\alpha}=\alpha^{p-1} \sum_{j k \cdots} Q_{i j k}\left(\frac{\partial^{p}}{\partial X_{j} \partial X_{k} \cdots} \frac{1}{R}\right)_{\mathbf{R}=r-\mathbf{R} n^{\alpha}}
$$

we evaluate in Appendix B the exact contributions to $\mathbf{S}_{1}, \mathbf{S}_{2}$, and $\mathbf{S}_{3}$ from all poles, considering only terms for which the resulting $\delta V_{e}$ is of first order in $q$. From (B2), (B3), and (B12) we obtain

$$
\begin{aligned}
& S_{1 i}=-\frac{8 \pi}{\Omega} i \sum_{j k l} Q_{i j k l} q_{j} \hat{q}_{k} \hat{q}_{l}, \\
& S_{3 i}=-\frac{8 \pi}{\Omega} \sum_{j k} Q_{i j k l} \hat{q}_{j} \hat{q}_{k}, \\
& S_{2 i}=\frac{8 \pi}{5 \Omega} i\left[2 \sum_{j k} Q_{i j k} q_{j} t_{k}-3 \sum_{j k l} Q_{i j k l} q_{j} \delta_{k l}\right],
\end{aligned}
$$

${ }^{20}$ In Ref. 2 it was stated that only even poles are admissible. It seems that there is no physical reason for this restriction. 
where $\Omega$ is the volume of the unit cell. We observe that only quadrupoles and octupoles are of interest to the present study. The total result for $\delta V_{e}$ is consequently

$$
\begin{aligned}
\delta V_{e}=A_{s} e^{i \mathrm{q} \cdot \mathrm{r}}(-8 \pi / \Omega) & \left\{\sum_{i j k} Q_{i j k}\left[q e_{1 i} \hat{q}_{j} \hat{q}_{k}+\frac{2}{5} i \hat{e}_{0 i} q_{j} t_{k}\right]\right. \\
& \left.+\sum_{i j k l} Q_{i j k l} i \hat{e}_{0 i} q_{j}\left[\hat{q}_{k} \hat{q}_{l}-\frac{3}{5} \delta_{k l}\right]\right\} .
\end{aligned}
$$

In cubic crystals, the symmetry yields that the quadrupole coefficients $Q_{i j k}$ are given in terms of a single parameter ${ }^{2} Q_{1}$ :

$$
Q_{i j k}=Q_{1}\left|\epsilon_{i j k}\right| \text {. }
$$

Likewise, we find for the octupole

$$
Q_{i j k l}=Q_{2} \delta_{i j} \delta_{j k} \delta_{k l} .
$$

Insertion of (3.15) and (3.16) in Eq. (3.14) leads to the final result

$$
\begin{aligned}
\delta V_{e}= & A_{s} e^{i \mathrm{q} \cdot \mathrm{r}} \\
& \times \sum_{i} i \hat{e}_{0 i}\left[C_{1} q_{i}+C_{2} \hat{q}_{i}{ }^{2} q_{i}+C_{3} \sum_{j k}\left|\epsilon_{i j k}\right| q_{j} t_{k}\right],
\end{aligned}
$$

with

$$
\begin{aligned}
& C_{1}=-(8 \pi / \Omega)\left[2 Q_{1} \gamma-(3 / 5) Q_{2}\right], \\
& C_{2}=(8 \pi / \Omega)\left[2 Q_{1} \gamma-Q_{2}\right], \\
& C_{3}=(16 \pi / 5 \Omega) Q_{1},
\end{aligned}
$$

and $\gamma$ has been introduced through the relation (2.20) between $\mathbf{e}_{1}$ and $\hat{e}_{0}$.

We have now obtained a nontrivial correction $\delta V_{e}$ to the deformation potential for acoustic-phonon scattering. The $C_{1}$ and $C_{2}$ terms in $\delta V_{e}$ are of the same form as the results found by Tolpygo ${ }^{3}$ from a different and less general model of the electrostatic fields. The $C_{3}$ term is new and results from the combined treatment of deformation and electrostatic potentials. The $C_{1}$ term is proportional to $\sum_{i j} \varepsilon_{i j}{ }^{\prime} \delta_{i j}=\operatorname{Tr} \varepsilon^{\prime}$ and may be added to the dilatational part of the deformation potential. However, it gives the same contribution to the conduction- and valence-band dilatational deformation-potential constants, and so the relation between these parameters via the static band-gap pressure coefficient is maintained in the dynamic case. The $C_{3}$ term contains the vector $\mathbf{t}$ which must be considered as a function of $\mathbf{r}$ having $\Gamma_{25}{ }^{\prime}$ symmetry. This is in accordance with the transformational properties required in Eq. (2.11) as well as with the fact that $\delta V_{e}$ should have cubic symmetry. It follows that the $C_{3}$ term is proportional to the off-diagonal elements of the strain tensor and will appear as a dynamic correction to the deformation-potential constants $d$ for degenerate valence bands ${ }^{11}$ and $\Xi_{u}$ for $\langle 111\rangle$ valleys. ${ }^{5}$ The remaining term with $C_{2}$ is of the form $\sum_{i j} \varepsilon_{i j}{ }^{\prime} \delta_{i j} \hat{q}_{i}{ }^{2}$ and has no resemblance to the deformation potential. Its inclusion in existing scattering theory is thus nontrivial and is discussed in Sec. IV.
Whenever electrostatic fields exist in the presence of free carriers, screening may be important. In the present case, the Debye screening length is always much larger than the phonon wavelength if phonons are the dominant scattering mechanism. We conclude that screening is not interesting in connection with phonon scattering.

An order-of-magnitude estimate of the long-range electrostatic effect may be obtained from lattice dynamics. ${ }^{2,3}$ In his theory of the phonon spectrum of Ge, $\mathrm{Lax}^{2}$ found the quadrupole-quadrupole interaction parameter $\rho=0.718 \times 10^{4} \mathrm{dyn} / \mathrm{cm}$. All higher-order poles were neglected, although their influence is largest near the zone boundary where the fit is most critical. ${ }^{2}$ Transforming into our notation, and taking into account the relative dielectric constant $\kappa_{r}=16$, we obtain

$$
Q_{1}^{2}=\frac{5}{6}\left(\frac{1}{4} a_{0}\right)^{5} \rho e^{2} / 4 \pi \kappa_{0} \kappa_{r},
$$

where $a_{0}$ is the lattice constant, $e$ the electronic charge, and $\left(4 \pi \kappa_{0}\right)^{-1} \approx 9 \times 10^{9} \mathrm{Nm}^{2} / \mathrm{C}^{2}$. From this we find

$$
(8 \pi / \Omega)\left|Q_{1}\right| a_{0}=4.3 \mathrm{eV} \text {. }
$$

The parameter $\gamma$ has been measured by Segmüller and Neyer. ${ }^{16}$ Defining the bond-bending parameter $\zeta_{0}$ by

$$
\gamma=-\frac{1}{8} a_{0} \zeta_{0},
$$

a value of $\zeta_{0}=0.64 \pm 0.04$ was found for Ge. ${ }^{16} \mathrm{Com}$ bining (3.22) and (3.23), we find from (3.19) that

$$
\left|C_{2}\right| \approx(8 \pi / \Omega) 2\left|Q_{1 \gamma}\right|=0.7 \mathrm{eV},
$$

where octupoles have been neglected. The same order of magnitude is found using Tolpygo's parameters, ${ }^{3}$ and similar results are obtained for $\mathrm{Si}^{3}$ We notice that $\left|C_{2}\right|$ is rather small compared to ordinary deformationpotential constants. The matrix elements of the $C_{3}$ term are difficult to estimate, since they depend on the wave functions of the band extrema. From (3.20) and (3.22), $\left|C_{3} t_{k}\right| \approx 0.2 \mathrm{eV}$, but the actual correction to $d$ and $\Xi_{u}(\langle 111\rangle$ valley) may be larger. Evidence about differences between static and dynamic values of these constants is not conclusive, but there exists indication of such an effect in $p$-Ge. ${ }^{1}$ Because of the largeness of $\Xi_{u}$ in $n$-Ge, ${ }^{6}$ experimental uncertainties in this parameter probably prohibit detection of the effect.

\section{ACOUSTIC SCATTERING IN Ge}

It is the purpose of this section to show that the nontrivial electrostatic correction to deformation-potential theory connected with the $C_{2}$ term in Eq. (3.17) can be included easily in existing scattering theory for the many-valley conduction-band structure ${ }^{5}$ as well as for the warped energy surfaces at the valence-band edge. ${ }^{1}$ Furthermore, we show that the correction to the transport parameters in $\mathrm{Ge}$ is negligible if $C_{2}$ is of the estimated magnitude.

For the $\langle 111\rangle$ valleys in $n$-Ge, the squared matrix element of the electron-phonon interaction in the 
deformation-potential approximation is given by

$$
\left.|M|^{2} \propto \sum_{s}\left[\delta E_{\langle 111}\right\rangle\left(\varepsilon_{s}^{\prime}(\mathbf{q})\right)\right]^{2} / \rho \omega_{s}(\mathbf{q})^{2},
$$

where $\rho$ is the mass density, $\omega_{s}(\mathbf{q})$ the frequency of an acoustic phonon with wave vector $\mathbf{q}$ in branch $s$, and

$$
\delta E_{\langle 111\rangle}\left(\varepsilon^{\prime}\right)=\left(\Xi_{d}+\frac{1}{3} \Xi_{u}\right) \varepsilon_{x x}{ }^{\prime}+\frac{2}{3} \Xi_{u} \varepsilon_{y z}{ }^{\prime}+\text { c.p. }
$$

Here, c.p. stands for cyclic permutation of $x, y$, and $z$, and

$$
\boldsymbol{\varepsilon}_{i j}^{\prime}=\frac{1}{2}\left(q_{i} \hat{e}_{0 j}+q_{j} \hat{e}_{0 i}\right),
$$

similar to Eq. (2.23). According to Eq. (3.17), the expression (4.2) should be extended to include the $C_{2}$ term in the following way:

$$
\begin{array}{r}
\delta E_{\langle 111\rangle}\left(\boldsymbol{\varepsilon}^{\prime}, \hat{q}\right) \\
\quad=\left(\Xi_{d}+\frac{1}{3} \Xi_{u}+C_{2} \hat{q}_{x}{ }^{2}\right) \varepsilon_{x x}{ }^{\prime}+\frac{2}{3} \Xi_{u} \varepsilon_{y z}{ }^{\prime}+\text { c.p. },
\end{array}
$$

whereas $C_{1}$ and $C_{3}$ are taken to be incorporated in $\Xi_{d}+\frac{1}{3} \boldsymbol{\Xi}_{u}$ and $\boldsymbol{\Xi}_{u}$, respectively.

In order to avoid unnecessary details we assume an isotropic acoustic-phonon spectrum with average longitudinal and transverse elastic constants $C_{l}$ and $C_{t}$ given by

$$
\begin{aligned}
& C_{l}=\frac{1}{5}\left(3 C_{11}+2 C_{12}+4 C_{44}\right), \\
& C_{t}=\frac{1}{5}\left(C_{11}-C_{12}+3 C_{44}\right) .
\end{aligned}
$$

The calculation of momentum-relaxation times then proceeds according to the method developed by Herring and Vogt. ${ }^{5}$ Using the notation of Ref. 5 where possible, we obtain for the squared effective deformation-potential constants ${ }^{5} \Xi_{\mathrm{II}^{2}}{ }^{2} \equiv[\|]$ and $\Xi_{\perp}{ }^{2} \equiv[\perp]$

$$
\Xi_{\nu}{ }^{2}=\Xi_{u}{ }^{2}\left[\xi_{\nu} r_{1}{ }^{2}+\eta_{\nu} r_{1}+\zeta_{\nu}+\xi_{\nu}{ }^{\prime} r_{2}{ }^{2}+\eta_{\nu}{ }^{\prime} r_{2}+\chi_{\nu}{ }^{\prime} r_{1} r_{2}\right],
$$

where $r_{1} \equiv \Xi_{d} / \Xi_{u}$ and $r_{2} \equiv C_{2} / \Xi_{u}$. The coefficients $\xi, \eta, \zeta$, $\xi^{\prime}, \eta^{\prime}$, and $\chi^{\prime}$ for parallel $(\|)$ and perpendicular $(\perp)$ directions are given in Table $I$ as function of the integrals ${ }^{5} I_{n}\left(\gamma_{m}\right)$ and $J_{n}\left(\gamma_{m}\right)$ and the ratios $\beta=C_{l} / C_{t}$ and $\gamma_{m}=m_{1} / m_{11}$.

From the original form (3.17), the $C_{2}$ term is expected to give rather anisotropic scattering. The anisotropy factor

$$
K_{\tau} \equiv \tau_{I 1} / \tau_{\perp}=\Xi_{\perp}^{2} / \Xi_{11}^{2}
$$

which is unity for isotropic scattering and about 1.20 for actual $n$-Ge, ${ }^{21}$ attains the value $3.3^{22}$ for $\Xi_{d}=\Xi_{u}=0$, thus confirming the expected anisotropy. On the other hand, for $\left|C_{2}\right| \approx 1 \mathrm{eV}$ and $\Xi_{u}=18 \mathrm{eV},{ }^{6}\left|r_{2}\right| \ll 1$, and so we conclude from (4.7) and Table I that the deformationpotential approximation is indeed very good for the transport theory of $n$-Ge. However, the "dynamic"

${ }^{21}$ C. Herring, T. H. Geballe, and J. E. Kunzler, Bell System Tech. J. 38, 657 (1959); L. J. Neuringer, in Proceedings of the Seventh International Conference on the Physics of Semiconductors Paris, 1964 (Academic Press Inc., New York, 1964), Vol. 1, p. 379.

\begin{tabular}{|c|c|c|c|}
\hline & $\|\left(I_{n}\right), \quad \perp\left(\frac{1}{2} J_{n}\right)$ & $\|$ & $\perp$ \\
\hline$\xi$ & $I_{0}$ & 1.33 & 1.33 \\
\hline$\eta$ & $2 I_{1}$ & 2.46 & 1.66 \\
\hline$\zeta$ & $I_{2}+\beta\left(I_{1}-I_{2}\right)$ & 1.29 & 1.09 \\
\hline$\xi^{\prime}$ & $-\frac{73}{12} I_{4}+20 I_{3}-\frac{45}{2} I_{2}+\frac{76}{9} I_{1}+\frac{1}{4} I_{0}$ & & \\
\hline & $+\beta\left[\frac{73}{12} I_{4}-\frac{1003}{48} I_{3}+\frac{185}{8} I_{2}-\frac{1201}{144} I_{1}+\frac{1}{18} I_{0}\right]$ & 0.14 & 0.46 \\
\hline$\eta^{\prime}$ & 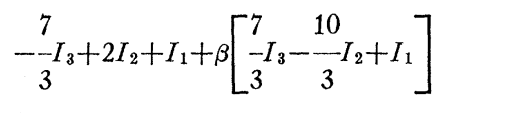 & 0.83 & 0.57 \\
\hline$x^{\prime}$ & $\stackrel{7}{-\frac{7}{3}}+2 I_{1}+I_{0}$ & 1.08 & 1.43 \\
\hline
\end{tabular}
${ }^{22}$ This value is subject to some uncertainty because the evaluation of $\xi^{\prime}$ in Table $I$ requires very high accuracy of $I_{n}$ and $J_{n}$. Since these integrals were read from Figs. 2 and 3 of Ref. 5 with an uncertainty of $2 \%$, the absolute error in $\xi^{\prime}$ is \pm 0.10 .
TABLE I. Relaxation-time coefficients for $\langle 111\rangle$ valleys in the theory of Herring and Vogt (Ref. 5) extended to include the $C_{2}$ term. The numerical values are calculated for $n$-Ge using $\gamma_{m}=0.05$ and $\beta=2.66$.

deformation-potential constants determined by comparison of theory and experiment are expected to be uncertain to the amount of $1 \mathrm{eV}$ because of the presence of the electrostatic effects. This is most important for the interpretation of the value of $\Xi_{d}+\frac{1}{3} \Xi_{u}$, which is rather small compared to $\Xi_{u \cdot}{ }^{6}$

For $p$-Ge, the existing theory ${ }^{1}$ can readily be extended to include the $C_{2}$ term of Eq. (3.17). In expressions (2.16) and (2.17) of Ref. 1 we just make the transformation

$$
a \varepsilon_{x x}^{\prime} \rightarrow\left(a-C_{2} \hat{q}_{x}^{2}\right) \varepsilon_{x x}{ }^{\prime},
$$

and similarly for $a \varepsilon_{y y}{ }^{\prime}$ and $a \varepsilon_{z z}{ }^{\prime}$. We notice the sign of $C_{2}$, which is a consequence of the fact that $C_{2}$ is measured as an electron energy, whereas the opposite sign convention was chosen in Ref. 1 for the valence-band deformation-potential constants. The inclusion of (4.9) in the calculations of Ref. 1 presents no computational difficulty. As in the case of $n$-Ge, the $C_{2}$ term introduces further anisotropy in the scattering. To illustrate this point, we have calculated the galvanomagnetic properties for the case where only $C_{2}$ is nonzero, i.e., $a=b=d=0$. The reduced, longitudinal magnetoresistance $^{1}$ in the $[100]$ direction then attained the anomalously large value of $b^{\prime}+c^{\prime}+d^{\prime}=0.55$ as compared to the value 0.058 for nearly isotropic scattering, $C_{2}=b$ $=d=0 .{ }^{1}$ However, for the more realistic case of $\left|C_{2}\right| \approx 1$ $\mathrm{eV}$, the change in galvanomagnetic properties (from the case with $C_{2}=0$ ) was found to be less than $5 \%$. This is in accordance with the simple isotropic momentum-relaxation-time model proposed in Ref. 1, where the variation of galvanomagnetic parameters is primarily due to a variable ratio of light- to heavy-hole relaxation times. We conclude that electrostatic effects may be neglected also in $p$-Ge, and that the deformation-potential approach gives a satisfactory picture of 
acoustic-phonon scattering. As we shall see presently, the electrostatic effects may be more important for the description of optical-phonon scattering.

\section{OPTICAL-PHONON SCATTERING}

Perhaps the most outstanding feature of the usual theory of nonpolar optical-phonon scattering is that this type of scattering is forbidden for certain band extrema as a consequence of symmety. ${ }^{9}$ Although generally claimed to be independent of the particular model used, this property will be disproved in the following.

The theory of optical-phonon scattering is based on the general interaction potential (2.1), and the displacements $\mathbf{u}_{n}{ }^{\alpha}$ are given by Eq. (2.18). For longwavelength optical phonons, the polarization vectors may be expanded in a power series in $q$, and to zeroth order we have

$$
\hat{e}^{\alpha}(\mathbf{q})=\alpha \hat{e}_{0}^{\prime}(\hat{q}) .
$$

Insertion in Eq. (2.1) then yields

$$
\delta V^{\prime}=A_{s}^{\prime} e^{i \mathrm{q} \cdot \mathrm{r}} \hat{e}_{0}{ }^{\prime}(\hat{q}) \cdot \sum_{n \alpha} \alpha \mathbf{V}_{n}{ }^{\alpha} e^{i \mathrm{q} \cdot\left(\mathrm{R}_{n} \alpha-\mathrm{r}\right)} .
$$

In the deformation-potential approximation, we assume the interaction-potential coefficients $\mathbf{V}_{n}{ }^{\alpha}$ to be of sufficiently short range so that a powers expansion in $\mathbf{q}$ of the sum in (5.2) exists. To zeroth order in $\mathbf{q}$ we then obtain

$$
\delta V_{d}^{\prime}=A_{s}^{\prime} e^{i \mathrm{q} \cdot \mathrm{r}} \hat{e}_{0}{ }^{\prime}(\hat{q}) \cdot \mathrm{V}_{2}(\mathbf{r})
$$

where $\mathrm{V}_{2}$ is given by Eq. (2.10). The optical deformation potential ${ }^{6,7} \mathbf{V}_{2}(\mathbf{r})$ has the symmetry of $\hat{e}_{0}{ }^{\prime}(\hat{q})$, i.e., $\Gamma_{25}{ }^{\prime}$ in cubic crystals. This gives the selection rule that the matrix element of $V_{2}$ vanishes for a nondegenerate band at $\mathbf{k}=0$ as well as for siliconlike minima on the cubic axes. ${ }^{9}$

As in the case of acoustic-phonon scattering, we now look at the long-range electrostatic contribution to the scattering potential. From the inversion-symmetry requirement (3.5) it is obvious that the electrostatic potential yields no direct contribution to $V_{2}$, and so the additional scattering potential $\delta V_{e}^{\prime}$ is given by $\mathrm{Eq}$. (5.2) with $\mathbf{V}_{n}{ }^{\alpha}$ standing for the multipole potentials (3.10). The sum in (5.2) is recognized as $\mathbf{S}_{3}$ defined in Eq. (3.9), and the result then follows from (3.12);

$$
\delta V_{e}^{\prime}=A_{s}{ }^{\prime} e^{i \mathrm{q} \cdot \mathrm{r}}\left(-\frac{8 \pi}{\Omega}\right) \sum_{i j k} Q_{i j k} \hat{e}_{0 i}{ }^{\prime} \hat{q}_{j} \hat{q}_{k} .
$$

Finally, we insert the cubic form (3.15) of $Q_{i j k}$ and obtain

$$
\delta V_{e}^{\prime}=A_{s}^{\prime} e^{i q \cdot r} C_{0} \frac{1}{2} \sum_{i j k}\left|\epsilon_{i j k}\right| \hat{e}_{0 i}{ }^{\prime} \hat{q}_{j} \hat{q}_{k}
$$

with

$$
C_{0}=-(16 \pi / \Omega) Q_{1}
$$

The total scattering potential is then

$$
\delta V^{\prime}=\delta V_{d}^{\prime}+\delta V_{e}^{\prime} .
$$

From the expression (5.5) for $\delta V_{e}^{\prime}$ we observe that the corresponding matrix element is always nonzero; in fact, it is independent of the band structure because $C_{0}$ is just a constant depending on the material. This is no violation of the cubic symmetry since the factor $\sum_{i j k}\left|\epsilon_{i j k}\right| \hat{e}_{0 i}{ }^{\prime} \hat{q}_{j} \hat{q}_{k}$ transforms as $\Gamma_{1}$ in $\mathbf{q}$ space.

The magnitude of $C_{0}$ in $\mathrm{Ge}$ can be estimated from Lax's theory of the phonon spectrum, ${ }^{2}$ and from (3.22) and (5.6) we find $\left|C_{0} a_{0}\right|=8.6 \mathrm{eV}$. Similar values are obtained from Tolpygo's theory ${ }^{3}$ for Ge and Si. For $p$-Ge this is to be compared with the value of the optical deformation-potential constant $d_{0} \approx 40 \mathrm{eV}$ inferred from the temperature dependence of the mobility. ${ }^{1}$ The corresponding constant for $n$-Ge is $D_{0} \approx 35$ $\mathrm{eV} .{ }^{23} \mathrm{It}$ is evident that the electrostatic contribution is a secondary effect if the "normal" part is nonvanishing. Nevertheless, it may have some influence in certain contexts because its form differs from that of the normal part. In $n-\mathrm{Si}$, where the normal part of the intravalley optical-phonon scattering vanishes by symmetry, ${ }^{9}$ the long-range electrostatic contribution is probably too weak compared to the strong $f$-type intervalley scattering between perpendicular valleys. ${ }^{10,24}$ Its importance may, however, be considerably enhanced under special experimental conditions, e.g., uniaxial stress, ${ }^{25}$ hot electrons, ${ }^{26}$ or both. ${ }^{27}$ The previously accepted model of scattering does not seem satisfactory in these cases. ${ }^{26-28}$

The transition rates for optical-phonon scattering modified by $\delta V_{e}^{\prime}$ are readily derived. For nondegenerate band extrema we find the following rate $P$ of transitions from $\mathbf{k}$ to $\mathbf{k}^{\prime}$ :

$$
\begin{aligned}
P\left(\mathbf{k}^{\prime}, \mathbf{k}\right)=\sum_{ \pm} \frac{\pi}{V \rho \omega_{0} a_{0}{ }^{2}}\left(n_{0}+\frac{1}{2} \pm \frac{1}{2}\right) & U^{0}\left(\mathbf{k}-\mathbf{k}^{\prime}\right) \\
& \times \delta\left(E^{\prime}-E \pm \hbar \omega_{0}\right),
\end{aligned}
$$

where $V$ is the volume of the crystal, $\rho$ is the mass density, $\omega_{0}$ the optical-phonon frequency at $\mathbf{q}=0, n_{0}$ the phonon occupation number at $\omega_{0}$, and $E$ and $E^{\prime}$ the energies corresponding to $\mathbf{k}$ and $\mathbf{k}^{\prime}$, respectively. $U^{0}$ is given by

and

$$
U^{0}(\mathbf{q})=\frac{1}{3}\left(D_{0}+D_{1} \hat{q}_{x} \hat{q}_{y}\right)^{2}+\text { c.p. }
$$

$$
D_{1}=C_{0} a_{0} \sqrt{3} \text {. }
$$

${ }^{23}$ M. H. Jørgensen, Phys. Rev. 156, 834 (1967). This value of $D_{0}$ is deduced from a theory of warm electrons, while the temperature dependence of the low-field mobility requires a $20 \%$ higher value (cf. Ref. 6).

${ }_{24}$ M. Lax and J. J. Hopfield, Phys. Rev. 124, 115 (1961).

${ }^{25} \mathrm{~J}$. E. Aubrey, W. Gubler, T. Henningsen, and S. H. Koenig, Phys. Rev. 130, 1667 (1963).

${ }_{26}$ M. Asche, B. L. Boitschenko, V. M. Bondar, and O. G. Sarbej, in Proceedings of the Eighth International Conference on the Physics of Semiconductors, Moscore, 1968 (Publishing House "Nauka", Leningrad, 1968), Vol. 2, p. 793.

${ }_{27}$ M. H. Jørgensen and N. I. Meyer, Solid State Commun. 3, 311 (1965).

${ }^{28} \mathrm{~J}$. C. McGroddy (private communication). 
For $\langle 100\rangle$ and $\langle 000\rangle$ valleys, $D_{0}=0$. Equation (5.9) exhibits the general feature that for $D_{1} \neq 0, P$ is not momentum-randomizing. ${ }^{5}$ It follows that this type of scattering cannot be treated analytically without rather drastic approximations in the solution of the Boltzmann transport equation.

For a degenerate extremum at $\mathbf{k}=0$, e.g., the valenceband edge, the transition rate from $\mathbf{k}$ in band $\lambda$ to $\mathbf{k}^{\prime}$ in band $\lambda^{\prime}$ may be expressed as in (5.8) with $U^{0}$ of the following form:

$$
\begin{aligned}
& U^{0}\left(\lambda^{\prime} \hat{k}, \lambda \hat{k}, \mathbf{q}=\mathbf{k}-\mathbf{k}^{\prime}\right) \\
& =\left(D d_{0} \hat{k}_{x} \hat{k}_{y} / E_{\hat{k} \lambda}+d_{1} \hat{q}_{x} \hat{q}_{y}\right)\left(D d_{0} \hat{k}_{x}{ }^{\prime} \hat{k}_{y}{ }^{\prime} / E_{\hat{k}^{\prime} \lambda^{\prime}}+d_{1} \hat{q}_{x} \hat{q}_{y}\right) \\
& +\left(\frac{1}{3} d_{0}{ }^{2}-d_{1}{ }^{2} \hat{q}_{x}{ }^{2} \hat{q}_{y}{ }^{2}\right) \Psi\left(\lambda^{\prime} \hat{k}^{\prime}, \lambda \hat{k}\right)+\text { c.p. }, \\
& d_{1}=-C_{0} a_{0} .
\end{aligned}
$$

For $d_{1}=0$, Eq. (5.11) reduces to Eq. (2.30) of Ref. 1, and the notation is the same as employed there. $\mathrm{Al}$ though (5.11) is not directly tractable when $d_{1} \neq 0$, some qualitative features will be brought out here in order to show that the electrostatic contribution may resolve one of the discrepancies between theory and experiment in $p$-Ge. ${ }^{1}$ In Ref. 1 it was shown that for galvanomagnetic effects, the acoustic-phonon scattering could be represented by isotropic relaxation times in the two bands. The Hall factor and transverse magnetoresistance depend quite critically on the ratio of light- to heavy-hole relaxation times. When $d_{1}=0$, the opticalphonon scattering can be represented by equal lightand heavy-hole relaxation times ${ }^{1}$ and this resulted in too high values of Hall factor and transverse magnetoresistance at room temperature. Qualitatively, the principal effect of the $d_{1}$ term is to render the opticalphonon relaxation times different in the two bands. If $d_{1}$ and $D d_{0}$ have opposite sign, the effect of the electrostatic correction will be to lower the above galvanomagnetic parameters at room temperature. Even modest values of $d_{1} / d_{0}$ may result in a considerable reduction without affecting the good agreement obtained for the temperature dependence of the mobility with $d_{1}=0 .{ }^{1}$

\section{NONLOCAL EFFECTS}

In Sec. II, we showed that infinitesimal-rotation invariance of the one-electron potential leads to the vanishing of the curlu term in the deformation potential (2.8). If the one-electron potential depends on properties of the electron other than position $\mathbf{r}$, the invariance is no longer present. An example of such a nonlocal effect is the spin-orbit coupling

$$
H_{\mathrm{so}} \propto\left(\nabla V_{0} \times \mathbf{p}\right) \cdot \boldsymbol{\sigma},
$$

where $\sigma_{x}, \sigma_{y}$, and $\sigma_{z}$ are the Pauli-spin matrices. Exchange effects ${ }^{29}$ are also of this nonlocal type. The spinorbit corrections to the deformation potential may be

${ }^{29}$ E. O. Kane, J. Phys. Chem. Solids 6, 238 (1958). evaluated by perturbation analysis, ${ }^{30}$ but once the effect has been established, there exists a more general method which is outlined below.

In an effective-mass representation following the displacement $\mathbf{u}_{1}$ (cf. Sec. II), the deformation potential assumes the form (for $\mathbf{k}=\mathbf{k}_{0}$ )

$$
\delta V_{d}^{\text {eff }}=\sum_{i j} D_{i j} \varepsilon_{i j}+\sum_{k} D_{k}{ }^{\prime} \operatorname{curl}_{k} \mathbf{u},
$$

which is a generalized version of Eq. (2.17). $\delta V_{d}^{\text {eff }}$, $D_{i j}$, and $D_{k}{ }^{\prime}$ are matrices of dimension corresponding to the degeneracy of the extremum at $\mathbf{k}_{0}$ including spin. In the related problem of $\mathbf{k} \cdot \mathbf{p}$ perturbation including a magnetic field, Luttinger ${ }^{31}$ showed that the form of the constant matrices $D_{i j}$ and $D_{k}{ }^{\prime}$ is determined by the symmetry of $\mathbf{k}_{0}$ and by the transformational properties of $\varepsilon$ and curlu relative to this symmetry. It is readily found that curlu transforms as an angular momentum. This leads to some interesting features which are brought out in the following simple example.

In the case of a simple spin-degenerate extremum at $\mathbf{k}=0$ in a cubic crystal, the effective deformation potential (6.2) has the form of a $2 \times 2$ matrix

$$
\delta V_{d^{\mathrm{eff}}}=\frac{1}{3} D \sigma^{2} \operatorname{Tr} \varepsilon+D^{\prime} \boldsymbol{\sigma} \cdot \text { curlu }
$$

where $D$ and $D^{\prime}$ are deformation-potential constants. If an arbitrary static deformation $\varepsilon$ and curlu is applied to the crystal, Eq. (6.3) shows that Tre gives a common shift of two spin bands, while curlu induces a splitting similar to the Zeeman effect. However, asymmetric deformations like curlu are not realized statically, but occur in combination with transverse phonons. For the squared matrix element of acoustic-phonon scattering, averaged over initial states and summed over final states with the same $\mathbf{k}$, we obtain from (6.3)

$$
|M|^{2}=D^{2}(\operatorname{Tr} \varepsilon)^{2}+D^{\prime 2}(\text { curlu })^{2} .
$$

We observe that there is no interference between the usual strain term and the new rotation term. This feature can be shown to be quite general for all types of band extrema exhibiting a Zeeman splitting.

For a transverse acoustic phonon, $(\operatorname{Tr} \varepsilon)^{2}$ and (curlu $)^{2}$ are of the same order of magnitude. If nonlocal effects should have any influence on the scattering, we must demand that $\left|D^{\prime}\right|$ is close to or larger than $|D|$, since the ratio of the two terms in (6.4) is quadratic in $D^{\prime} / D$. We expect, however, that spin-orbit corrections and exchange effects are relatively small in $\mathrm{Si}$ and $\mathrm{Ge},{ }^{32}$ and so nonlocal effects have no practical importance for phonon scattering and are merely of academic interest.

\section{DISCUSSION}

In this work, we have shown that the deformationpotential approach to long-wavelength one-phonon

${ }^{30}$ P. Lawaetz, thesis, The Technical University of Denmark, Lyngby, Denmark (unpublished).

31 J. M. Luttinger, Phys. Rev. 102, 1030 (1956)

${ }^{32}$ M. Cardona and F. H. Pollak, Phys. Rev. 142, 530 (1966). 
scattering in nonpolar semiconductors is subject to nontrivial corrections arising from long-range electrostatic forces. ${ }^{2,3}$ However, these corrections are found to be very small, and have no influence on the transport properties except for cases where a nontrivial correction to the usual description of optical-phonon scattering may be important, i.e., in $p$-Ge and $p$-Si as well as in $n$-Si under special experimental conditions. It would be natural to inquire whether there is any general physical reason for the smallness of these effects. Since we shall make no attempt to look into details of the potentials involved, our answer to this question is hardly more than a suggestion.

In the theory of phonon spectra in nonpolar semiconductors, $\operatorname{Lax}^{2}$ found a qualitatively satisfactory model consisting of nearest-neighbor force constants augmented by long-range quadrupole-quadrupole interactions. The long-range electrostatic forces are needed to fit the experimental phonon frequencies near the zone edges and have but little effect near $\mathbf{q}=0$. This suggests that the forces involved are mostly of short range and do not extend appreciably outside the unit cell. The electrostatic forces are comparable in magnitude to next-neighbor forces and are important only because of their nontrivial behavior at large $\mathbf{q}$.

It is tempting to suppose that the same applies to the change in the one-electron potential caused by the deformation of the lattice, i.e., the scattering potential. This means that the electron at $\mathbf{r}$ perceives almost only the displacement of the two nearest atoms, that is, those inside the cell containing $\mathbf{r}$. Thus, the effect of farther-away atomic displacements is small and so the electrostatic interaction, which actually belongs to this category, ${ }^{33}$ has the same property. It follows that if the long-range electrostatic effects introduce no singular behavior of the scattering potential for long wavelengths, they will have a very small effect on scattering in this region. As discussed in this paper, singular features only occur in practice in connection with optical-phonon scattering. We conclude that this model is in qualitative agreement with the general results of the present work.

If the model is essentially correct, it may offer an important simplification in the treatment of interactions involving short-wavelength phonons. By relatively simple means one may thus estimate the strength of allowed ${ }^{24}$ intervalley transitions from a knowledge of deformation-potential constants pertaining to longwavelength phonon scattering. This and other features of the short-range model will be the subject of further investigations.

\section{ACKNOWLEDGMENTS}

The author is indebted to Professor N. I. Meyer for his continued encouragement throughout the course of

\footnotetext{
${ }^{33}$ It was shown in Sec. III that the multipoles at $n=0$ are determined by the poles for $n \neq 0$ by means of the invariance properties (3.3) and (3.5).
}

this work. Helpful discussions with Dr. M. H. Jørgensen are also gratefully acknowledged.

\section{APPENDIX A: INFINITESIMAL-DISPLACEMENT INVARIANCE AND INVERSION SYMMETRY}

For a $2^{p}$-pole at $\mathbf{R}_{n}{ }^{\alpha}$, the potential at $\mathbf{r}$ is of the form shown in Eq. (3.10). To evaluate the sums (3.3) and (3.5) we shall use the Fourier transform

$$
\frac{1}{R}=\frac{1}{2 \pi^{2}} \int d^{3} K K^{-2} e^{-i \mathrm{~K} \cdot \mathrm{R}}
$$

and the relation

$$
\sum_{n} e^{i \mathbf{K} \cdot\left(\mathbf{R}_{n}-\mathbf{R}_{0}\right)}=\frac{(2 \pi)^{3}}{\Omega} \delta(\mathbf{K}),
$$

where $\Omega$ is the volume of the unit cell. In terms of its Fourier transform, $\mathbf{V}_{n}{ }^{\alpha}$ has the following form:

$$
\begin{array}{r}
V_{n i}^{\alpha}=\frac{1}{2 \pi^{2}}(-i)^{p} \sum_{j k \ldots} Q_{i j k} \ldots \int d^{3} K K^{-2} K_{j} K_{k} \ldots \alpha^{p-1} \\
\\
\times e^{-i \mathbf{K} \cdot\left(\mathbf{r}-\mathbf{R}_{0}-\alpha \mathrm{t}\right)} e^{i \mathbf{K} \cdot\left(\mathbf{R}_{n}-\mathbf{R}_{0}\right)} .
\end{array}
$$

By means of (A2) we then find

$$
\begin{array}{r}
\sum_{n} V_{n i}{ }^{\alpha}=\frac{4 \pi}{\Omega}(-i)^{p} \sum_{j k \ldots} Q_{i j k} \ldots \int d^{3} K K^{-2} K_{j} K_{k} \ldots \delta(\mathbf{K}) \\
\times \alpha^{p-1} e^{-i \mathbf{K} \cdot\left(\mathbf{r}-\mathbf{R}_{0}-\alpha \mathrm{t}\right)} .
\end{array}
$$

We see immediately that for $p>2, \sum_{n}=0$ independent of $\alpha$. For $p=2$, we sum (A4) over $\alpha$ to obtain

$$
\begin{aligned}
\sum_{n \alpha} V_{n i}{ }^{\alpha} & =-\frac{8 \pi i}{\Omega} \sum_{j k} Q_{i j k} \int d^{3} K K^{-2} K_{j} K_{k} \sin (\mathbf{K} \cdot \mathbf{t}) \\
& =0 .
\end{aligned}
$$

We have thus shown that all poles with $p \geq 2$ satisfy the requirement (3.3) of the infinitesimal-displacement invariance, provided that the potential arising from the displacement of the two atoms with $n=0$ is described by the same poles as for $n \neq 0$.

For $p>2$ we have already shown in (A4) that $\sum_{n \alpha} \alpha V_{n}^{\alpha}=0$, whereas for $p=2$,

$$
\sum_{n \boldsymbol{\alpha}} \alpha V_{n i}{ }^{\alpha} \propto \sum_{j k} Q_{i j k} \delta_{j k} .
$$

According to the macroscopic inversion symmetry (3.5), the sum in (A6) must vanish, and so

$$
\sum_{j k} Q_{i j k} \delta_{j k}=0
$$

must be satisfied by the quadrupole. In the cubic case (diamond lattice), $Q_{i j k}$ is of the form (3.15), so that the requirement (A7) is automatically fulfilled. 


\section{APPENDIX B : SUMMATION OF THE ELECTROSTATIC TERMS}

In this Appendix we evaluate the sums $\mathbf{S}_{1}, \mathbf{S}_{2}$, and $\mathbf{S}_{3}$ given by Eqs. (3.7)-(3.9), respectively, using the multipole potential $\mathbf{V}_{n}{ }^{\alpha}$ from (3.10). Its Fourier transform is shown in Eq. (A3). On applying the summation relation (A2) we have

$$
\begin{aligned}
\sum_{n} V_{n i}{ }^{\alpha} e^{i \mathrm{q} \cdot\left(\mathbf{R}_{n}{ }^{\alpha-\mathbf{r})}=\right.}=\frac{4 \pi}{\Omega}-\alpha^{p-1} i^{p} & \\
& \times \sum_{j k \ldots} Q_{i j k \ldots} \ldots q^{-2} q_{j} q_{k} \cdots .
\end{aligned}
$$

It is then obvious that $\mathbf{S}_{1}$ contains only odd poles, whereas $\mathbf{S}_{3}$ has contributions only from even poles. To first order in $q$ we obtain

and

$$
S_{1 i}=-\frac{8 \pi}{\Omega} i \sum_{j k l} Q_{i j k l} q_{j} \hat{q}_{k} \hat{q}_{l}
$$

$$
S_{3 i}=-\frac{8 \pi}{\Omega} \sum_{j k} Q_{i j k} \hat{q}_{j} \hat{q}_{k}
$$

In order to evaluate $\mathbf{S}_{2}$, we introduce an artificial oscillatory variation with wave vector $\boldsymbol{\kappa}$ :

$$
\mathbf{V}_{n}^{\alpha}=\lim _{\kappa \rightarrow 0} \mathbf{V}_{n}^{\alpha} e^{-i \kappa \cdot\left(\mathbf{R}_{n}-\mathbf{R}_{0}\right)}
$$

On taking the limit $\boldsymbol{\kappa} \rightarrow 0$, we should only consider the isotropic (principal) part of the argument in $\boldsymbol{\kappa}$ space. Actually, the form (B4) is also used to derive Eq. (A6) Then from (3.8)

$$
S_{2 i}=-\sum_{n \alpha \lambda} q_{\lambda} V_{n i}{ }^{\alpha} \lim _{\kappa \rightarrow 0} \frac{\partial}{\partial \kappa_{\lambda}} e^{-i \kappa \cdot\left(\mathbf{R}_{n}-\mathbf{R}_{0}\right)} .
$$

Insertion of the Fourier transform of $V_{n i}{ }^{\alpha}$ gives

$$
\begin{array}{r}
S_{2 i}=-\sum_{\alpha \lambda} \sum_{j k \ldots} q_{\lambda} \frac{4 \pi}{\Omega} Q_{i j k} \ldots \alpha^{p-1}(-i)^{p} \lim _{\kappa \rightarrow 0} \int d^{3} K K^{-2} \\
\times K_{j} K_{k \ldots} \ldots e^{-i \mathbf{K} \cdot\left(\mathbf{r}-\mathbf{R}_{0}-\alpha \mathrm{t}\right)} \frac{\partial}{\partial \kappa_{\lambda}} \delta(\mathbf{K}-\boldsymbol{\kappa}),
\end{array}
$$

and so by means of a well-known $\delta$-function theorem

$$
\begin{array}{r}
S_{2 i}=-\frac{4 \pi}{\Omega}(-i)^{p} \sum_{\alpha \lambda} q_{\lambda} \alpha^{p-1} \sum_{j k \cdots} Q_{i j k} \ldots \lim _{\kappa \rightarrow 0} \frac{\partial}{\partial \kappa_{\lambda}} \\
\times\left[\kappa^{-2} \kappa_{j} \kappa_{k} \cdots e^{-i \kappa \cdot\left(\mathbf{r}-\mathbf{R}_{0}-\alpha \mathrm{t}\right)}\right] .
\end{array}
$$

It is immediately seen that this vanishes for $p \geq 4$. For $p=2$ we find

$$
\begin{aligned}
S_{2 i}{ }^{\prime}= & \frac{8 \pi i}{\Omega} \sum_{j k \lambda \mu} q_{\lambda} t_{\mu} Q_{i j k} \lim _{\kappa \rightarrow 0}\left\{-2 \hat{\kappa}_{\lambda} \hat{\kappa}_{j} \hat{\kappa}_{k} \hat{\kappa}_{\mu}\right. \\
& \left.+\hat{\kappa}_{j} \hat{\kappa}_{k} \delta_{\mu \lambda}+\hat{\kappa}_{j} \hat{\kappa}_{\mu} \delta_{\lambda k}+\hat{\kappa}_{k} \hat{\kappa}_{\mu} \delta_{\lambda j}\right\} \\
= & \frac{8 \pi i}{5 \Omega} \sum_{j k} Q_{i j k}\left[q_{j} t_{k}+q_{k} t_{j}+(\mathbf{q} \cdot \mathbf{t}) \delta_{j k}\right] .
\end{aligned}
$$

But according to Eq. (A7) the last term vanishes, and because $Q_{i j k}$ is symmetric in $j$ and $k$, we find

$$
S_{2 i}^{\prime}=\frac{16 \pi i}{5 \Omega} \sum_{j k} Q_{i j k} q_{j} t_{k}
$$

For $p=3$ we obtain from (B7)

$$
\begin{aligned}
S_{2 i}^{\prime \prime}= & -\frac{8 \pi i}{\Omega} \sum_{\lambda j k l} Q_{i j k l} q_{\lambda} \lim _{\kappa \rightarrow 0}\left\{-2 \hat{\kappa}_{j} \hat{\kappa}_{k} \hat{\kappa}_{l} \hat{\kappa}_{\lambda}\right. \\
\left.+\hat{\kappa}_{j} \hat{\kappa}_{k} \delta_{\lambda l}+\hat{\kappa}_{k} \hat{\kappa}_{l} \delta_{\lambda j}+\hat{\kappa}_{l} \hat{\kappa}_{j} \delta_{\lambda k}\right\} & \\
= & -\frac{8 \pi i}{5 \Omega} \sum_{j k l} Q_{i j k l}\left[q_{j} \delta_{k l}+q_{k} \delta_{l j}+q_{l} \delta_{j k}\right]
\end{aligned}
$$

and using the interchangeability of $j, k$, and $l$, we arrive at

$$
S_{2 i}^{\prime \prime}=-\frac{24 \pi i}{5 \Omega} \sum_{j k l} Q_{i j k l} q_{j} \delta_{k l}
$$

Finally, the total $S_{2 i}$ is found by addition of (B9) and (B11):

$$
S_{2 i}=\frac{8 \pi}{5 \Omega} i\left[2 \sum_{j k} Q_{i j k} q_{j} t_{k}-3 \sum_{j k l} Q_{i j k l} q_{j} \delta_{l k l}\right] .
$$

